\title{
Electron crystallography of molecular crystals
}

\author{
L. Palatinus \\ Institute of Physics of the Czech Academy of Sciences, Na Slovance 2, 18221 Prague 8, Czechia \\ palat@fzu.cz
}

Electron diffraction is gradually becoming a broadly accepted alternative structure determination method to the established approaches like single crystal x-ray diffraction. Although electron diffraction was used for structure determination long time ago, the real breakthrough was obtained only thanks to the rapid development of the three-dimensional electron diffraction methods (3D ED). Several 3D ED experimental techniques exist, but they all share the basic principle of collecting three-dimensional diffraction information from a single very small crystal by tilting the crystal, continuously or in steps, and recording the diffraction patterns as the crystal is tilted [1].

In the early days of 3D ED, mostly inorganic materials were analysed, as these materials are less beam sensitive and therefore easier to measure. Nevertheless, soon first successful attempts to analyse organic materials appeared (reference). Gradually, over the past ten years, structure analysis of organic materials by $3 \mathrm{D}$ ED has become almost a routine technique.

Structure analysis of molecular materials by 3D ED has certain specifics. First, molecular crystals are, typically, much more beam sensitive than crystals of inorganic materials, although this rule is by far not strictly applicable. The beam sensitivity of organic materials calls for specific experimental techniques. These include: measurements at low-temperature conditions (usually cooling to liquid nitrogen temperatures) to limit the beam damage; use of modern direct electron detectors with low background and high sensitivity to maximize the signal-to-noise ratio; combination of partial data sets from several crystals; or application of serial diffraction techniques. The latter can include either scanning a large single crystal with a small probe, measuring each diffraction pattern on a fresh part of the crystal [2,3], or collecting single diffraction patterns from a large number of crystals and combining them in a manner similar to serial femtosecond crystallography [4].

Once suitable data are collected, the challenge continues in the structure analysis part. It is usually not complicated to solve the structure by ab initio structure solution methods. However, it is challenging to obtain a good quality refinement. The multiple scattering effects that are always present in electron diffraction break the kinematical nature of the diffraction. If the kinematical approximation is used in the refinement, the figures of merit of the refinement tend to be high and weak signals in the structure may be obscured. This makes, for example, the detection of hydrogen atoms difficult, although not impossible $[5,6]$. The fit to experimental data can be improved by employing the dynamical diffraction theory in the calculation of the model intensities [3, 6, 7]. Such calculation results in an improved fit to experimental data and, as a consequence, to higher accuracy of the refined parameters and better sensitivity to weak features. A very important advantage of using the dynamical diffraction theory in the refinement is the possibility to determine the absolute structure of the crystals [3].

Despite of al the challenges, 3D ED was already proved to be an extremely successful technique for structure determination of molecular crystals. The fact that it can routinely analyse crystals with sub-micrometre size makes it an attractive alternative to single crystal x-ray diffraction.

[1] Gemmi, M., Mugnaioli, E., Gorelik, T. E., Kolb, U., Palatinus, L., Boullay, P., Hovmöller, S., Abrahams J. P. (2019). ACS Cent. Sci. 5, 1315.

[2] Kolb, u., Mugnaioli, E., Gorelik, T. (2011). Cryst. Res. Technol. 46, 542.

[3] Brázda, P., Palatinus, L., Babor, M. (2019). Science 364, 667

[4] Smeets, S., Zou, X., Wan, W. (2018). J Appl Cryst. 51, 1262.

[5] Jones, C. G., Martynowycz, M. W., Hattne,, J. Fulton,T. J., Stoltz, B. M., Rodriguez, J. A., Nelson, H. M., Gonen, T. (2018). ACS Cent Sci. 4, 1587.

[6] Palatinus, L., Brázda, P, Boullay, P., Perez, O., Klementová, M., Petit, S., Eigner, V., Zaarour, M, Mintova, S. (2017). Science 355, 166.

[7] Palatinus, L., Petricek, V., Correa, C. A. (2015). Acta Cryst. A 71, 235.

Keywords: 3D electron diffraction; structure refinement; nanocrystals; absolute structure

This work was funded by the Czech Science Foundation, project number 21-05926X. 